\title{
Weak Peristalsis in Esophageal Pressure Topography: Classification and Association With Dysphagia
}

\author{
Sabine Roman, MD, PhD ${ }^{1}$, Zhiyue Lin, MS ${ }^{1}$, Monika A. Kwiatek, PhD ${ }^{1}$, John E. Pandolfino, \\ MD ${ }^{1}$, and Peter J. Kahrilas, MD ${ }^{1}$ \\ ${ }^{1}$ Department of Medicine, The Feinberg School of Medicine, Northwestern University, Chicago, \\ Illinois, USA
}

\section{Abstract}

OBJECTIVES-Esophageal manometry is frequently used to assess for weak peristalsis. Although commonly used clinically, there are currently no validated metrics of weak peristalsis in high-resolution esophageal pressure topography (EPT). This study aimed to develop a classification of weak peristalsis in EPT based on a comparative analysis of control subjects and patients with unexplained non-obstructive dysphagia.

METHODS-High-resolution esophageal pressure topography (high-resolution impedance manometry) studies were carried out in 16 control subjects to verify EPT features associated with incomplete bolus transit (IBT). The technique of superimposing EPT plots in a computer simulation was used to derive normal limits of peristaltic integrity in EPT in another 75 control subjects. The occurrence of critical EPT defects was then compared between control subjects and 113 patients with non-obstructive dysphagia identified from a large clinical series.

RESULTS-IBT occurred with failed peristalsis or with breaks in the $20 \mathrm{~mm} \mathrm{Hg}$ isobaric contour occurring at the proximal or distal pressure troughs in EPT plots. The normal range for isobaric contour breaks was $0-20 \%$ for large (> $5 \mathrm{~cm})$ and $0-30 \%$ for small $(2-5 \mathrm{~cm})$ breaks, with both occurring significantly more frequently in dysphagic patients. Failed peristalsis was not more frequent in dysphagic patients.

CONCLUSIONS-A classification of weak peristalsis adapted to EPT is proposed based on the occurrence of breaks in the $20 \mathrm{~mm} \mathrm{Hg}$ isobaric contour wherein weak peristalsis with large breaks is defined by those occurring with $>20 \%$ of swallows and weak peristalsis with small breaks defined by those occurring with $>30 \%$ of swallows.

\section{INTRODUCTION}

One of the main clinical applications of esophageal manometry is to assess the integrity of peristalsis, either as part of an evaluation of dysphagia or in anticipation of antireflux surgery. Conventionally, this has been carried out by measuring the amplitude of the peristaltic contraction at arbitrary locations within the distal esophagus (1). The most

Correspondence: Peter J. Kahrilas, MD, Division of Gastroenterology, Department of Medicine, Feinberg School of Medicine, Northwestern University, 676 St Clair Street, Suite 1400, Chicago, Illinois 60611-2951, USA. p-kahrilas@ northwestern.edu.

Guarantor of the article: Peter J. Kahrilas, MD.

Specific author contributions: Analysis and interpretation of data and drafting of the paper: Sabine Roman; analysis and interpretation of data: Zhiyue Lin and Monika A. Kwiatek; study concept and design, analysis and interpretation of data, and drafting of the paper: John E. Pandolfino and Peter J. Kahrilas.

CONFLICT OF INTEREST

Potential competing interests: None. 
commonly accepted metrics establishing normality are peristaltic amplitude exceeding 30 $\mathrm{mm} \mathrm{Hg}$ at 3 and $8 \mathrm{~cm}$ proximal to the lower esophageal sphincter (LES) (2) based on the observation that amplitudes $<30 \mathrm{~mm} \mathrm{Hg}$ are frequently associated with bolus escape and incomplete bolus clearance (3). However, with the evolution of two technologies, intraluminal impedance monitoring (4) and high-resolution esophageal pressure topography (EPT) plotting (5), it has become apparent that these conventional metrics provide a very incomplete assessment of peristaltic integrity. Findings from multichannel intraluminal impedance recordings suggest that the $30 \mathrm{~mm} \mathrm{Hg}$ threshold value is too high in many instances (6), whereas EPT studies suggest that the arbitrary selection of two foci to measure pressure amplitude ignores much of the detail and variability inherent in the peristaltic contraction that are relevant to normal function (7).

Compounding the technical limitations of conventional manometry in the assessment of peristaltic integrity, there is the added liability that this has always been a poorly standardized clinical test $(8,9)$. The number and locations of pressure sensors as well as the quantitative limits of normality in terms of how many weak contractions are acceptable for the definition are not standardized. Each of these limitations is potentially addressed by the combination of EPT and intraluminal impedance monitoring; impedance monitoring provides a method for verifying complete or incomplete bolus transit (IBT) and EPT can provide a complete description of the associated intraluminal contractility. We previously used high-resolution impedance manometry (HRIM) in a pilot study of 16 control subjects and 8 patients with dysphagia to analyze the correlation between EPT features and IBT (10). That study showed that peristaltic contractions with an intact $20 \mathrm{~mm} \mathrm{Hg}$ isobaric contour in the EPT plot were invariably associated with complete bolus transit and IBT could be attributed to failed peristalsis or breaks in the $20 \mathrm{~mm} \mathrm{Hg}$ isobaric contour. However, no systematic analysis has yet been done in a larger series of control subjects and patients to provide guidance on appropriate EPT metrics of peristaltic integrity in clinical studies. Consequently, the aims of this investigation were (i) to establish normal limits for defining peristaltic integrity based on a detailed, systematic analysis of a large series of normal control subjects; (ii) to test for abnormalities of these metrics in a cohort of patients with unexplained non-obstructive dysphagia; and (iii) to develop a classification scheme for weak peristalsis in EPT terms intended for use in clinical EPT studies.

\section{METHODS}

\section{Subjects and study protocol}

HRIM studies were carried out in 18 control subjects, but owing to technical failures, data were available in only 16 ( 7 males, mean age 33 years, range 20-50). High-resolution manometry (HRM) studies were carried out in 75 asymptomatic subjects (40 males, mean age 27 years, range 19-48 years). Volunteers were recruited by advertisement or word of mouth and had no history of gastro-intestinal symptoms or surgery. The study protocol was approved by the Northwestern University Institutional Review Board and informed consent was obtained from each subject. Dysphagic patients were selected from a consecutive series of 2,000 patients after excluding individuals with major motility disorders by the Chicago Classification criteria (11) (achalasia, absent peristalsis, diffuse esophageal spasm, spastic nutcracker, functional obstruction defined by an elevated integrated relaxation pressure (IRP) with some preserved peristalsis), hiatus hernia ( $\geq 2 \mathrm{~cm}$ separation between the lower esophageal sphincter and crural diaphragm), esophageal stricture, or eosino philic esophagitis (endoscopic or histopathological evidence). In all, 113 patients (37 males, mean age 46 years, range 21-76) met these criteria of unexplained dysphagia.

Manometric studies were carried out with the patients in supine position after at least a 6-h fast. Both the HRIM and HRM catheters were $4.2 \mathrm{~mm}$ outer diameter solid-state assemblies 
with 36 circumferential sensors at 1-cm intervals (Sierra Scientific Instruments, Los Angeles, CA). The HRIM catheter also incorporated 18 impedance segments at 2-cm intervals. Transducers were calibrated at 0 and $300 \mathrm{~mm} \mathrm{Hg}$ using externally applied pressure. The manometry assemblies were placed transnasally and positioned to record from the hypopharynx to the stomach with about three intragastric sensors. The manometric protocol included a 5-min baseline recording and ten 5-ml swallows. The study protocol for all HRM and HRIM studies was identical with the caveat that saline, not water, was used for test swallows in HRIM studies.

\section{HRIM analysis}

The EPT of each swallow in the HRIM studies was systematically analyzed for integrity of the 20 and $30 \mathrm{~mm} \mathrm{Hg}$ isobaric contours. EPT was defined as intact if no break was observed in the isobaric contour. Instances in which no peristaltic contraction followed a swallow were characterized as failed peristalsis. When the 20 or the $30 \mathrm{~mm} \mathrm{Hg}$ isobaric contour was disrupted, the length of the break was measured using the Smart Mouse tool in ManoView software (Sierra Scientific Instruments). Contractile Front Velocity (CFV), the speed at which the propagated contraction progresses along the esophagus, was also measured. This was estimated as the slope of a tangent drawn to skirt, but not intersect, the $30 \mathrm{~mm} \mathrm{Hg}$ isobaric contour of the contractile segment between the proximal pressure trough and the contractile deceleration point as previously described (12).

HRIM studies were then analyses to define EPT features associated with IBT. Bolus transit was assessed using the impedance tracing and colorized contour functions of ManoView. Colorization was adjusted to the threshold at which pink colorization corresponded to bolus presence on impedance tracings (Figure 1). Bolus transit was considered complete if colorization was followed by color disappearance and incomplete if this was not the case. Impedance tracings of all instances of IBT were reviewed to verify that they failed to meet established criteria for complete bolus transit ( $\geq 50 \%$ decrease in impedance) (4).

\section{Characterizing EPT from normalized swallows}

In order to characterize EPT among control subjects, the timing of peristalsis relative to the swallow had to be synchronized and the length of the esophagus from sphincter to sphincter had to be normalized. This was done with exported Manoscan ${ }^{\mathrm{TM}}$ data using a customized MATLAB $^{\text {TM }}$ program (The Math Works, Natick, MA) to create a $100 \times 200$ pixel coordinate-based scheme that standardized the length of the esophagus as 100 pixels (0-100 $\%$ spanning from the last sensor in the pharynx to the first sensor in the stomach) and time as 200 pixels ( $0.1 \mathrm{~s}$ increments from $1 \mathrm{~s}$ before the onset of UES relaxation (to $<20 \mathrm{~mm} \mathrm{Hg}$ ) until after $19 \mathrm{~s}$ (13). Pressure within each pixel was then analyzed during peristalsis. A 10swallow composite was first computed for each subject based on median pixel pressures during peristalsis among swallows. Instances of failed peristalsis were not included; in those instances, fewer than 10 swallows were used to construct the composite EPT. The 75 individual composites were then combined to compute the median, 25th, and 5th percentile simulations.

\section{Comparison of EPT integrity in control subjects and dysphagia patients}

The normal range for EPT features associated with IBT was established by scoring each of the 75 control subject's EPT study for the occurrence of features identified from the HRIM findings and EPT simulation. Statistical norms were derived from the composite picture of defect type and distribution and these were compared with findings in the dysphagia group. As with the control subjects, each dysphagia patient's EPT was scored for the frequency of defects potentially associated with IBT. 


\section{Statistics}

Descriptive statistics (median, 5th, 95th percentiles) were used in control subjects to describe manometric findings as they had a non-parametric distribution. In dysphagia patients, data were described as median (interquartile range). Data between groups were compared using the $\chi^{2}$-test for categorical data and Mann-Whitney test for continuous data. A $P$ value $<0.05$ was considered significant.

\section{RESULTS}

\section{High-resolution impedance manometry}

HRIM data were evaluable in 16 control subjects. Complete bolus transit in all test swallows was seen in $6(38 \%)$ of these and the median percentage of swallows with IBT among the 16 subjects was $15 \%$ (interquartile range $0-33 \%$ ). Seven (44\%) subjects exhibited one or more instances of failed peristalsis (total of 13 swallow sequences) and these were uniformly associated with IBT.

The EPT plots in the 14 instances of IBT not attributable to failed peristalsis were all associated with breaks in the $20 \mathrm{~mm} \mathrm{Hg}$ isobaric contour at the proximal or distal pressure troughs. In characterizing these breaks, Figure 2 illustrates that, although breaks could be scored in either the 20 or $30 \mathrm{~mm} \mathrm{Hg}$ isobaric contour, optimal discrimination between complete and IBT was achieved using the $20 \mathrm{~mm} \mathrm{Hg}$ isobaric contour. Consequently, this convention was adopted. IBT never occurred in the absence of breaks in the $20 \mathrm{~mm} \mathrm{Hg}$ isobaric contour. Table 1 summarizes the peristaltic defects associated with IBT; in instances in which more than one isobaric contour break occurred, only the more proximal break with impedance evidence of IBT was scored. Based on these data, breaks in the 20 $\mathrm{mm} \mathrm{Hg}$ isobaric contour were categorized as large, small, or insignificant, depending on their length. Large (> $5 \mathrm{~cm}$ ) breaks were uniformly associated with IBT (sensitivity $71 \%$, specificity $100 \%)$. Small $(2-5 \mathrm{~cm})$ breaks were associated with IBT in only $16 \%$ of instances (sensitivity $100 \%$, specificity $84 \%$ ). Figure 3 illustrates examples of IBT attributable to large and small breaks in the $20 \mathrm{~mm} \mathrm{Hg}$ isobaric contour.

Although breaks in the $20 \mathrm{~mm} \mathrm{Hg}$ isobaric contour had a significant relationship to IBT, the CFV did not. Figure 4 illustrates two examples of EPT plots with high CFV, one with an associated isobaric contour break and the other not. Consistent with these examples, of the 10 instances of high CFV (>10 cm/s) among subjects, the only instance of associated IBT was also associated with a large break in the $20 \mathrm{~mm} \mathrm{Hg}$ isobaric contour.

\section{Normalized EPT of peristalsis}

The process of generating a composite of all intact peristaltic contractions for each subject had a smoothing effect on the resultant EPT plot, nearly eliminating pressure fluctuations attributable to respiration or heartbeat that were not synchronized with swallowing (13). Normalizing the composite EPT plots of the entire group of 75 control subjects had the further effect of extracting the stereotyped features of peristalsis common to the entire group. Data within each pixel of the $100 \times 200$ normalized grid are summarized in Figure 5 illustrating the median, 25th, and 5th percentiles of the peristaltic contraction in EPT format for all 75 volunteer subjects. Two pressure troughs are clearly identified in each panel of Figure 5, one proximal (P) and one distal (D). Note that, although the $20 \mathrm{~mm} \mathrm{Hg}$ isobaric contour is seamless in the median EPT plot (Figure 5, top panel), this exhibits increasingly larger breaks at $\mathrm{P}$ and $\mathrm{D}$ in the 25th and 5th percentile composite EPT plots, respectively. The median length of $20 \mathrm{~mm} \mathrm{Hg}$ isobaric contour break at $\mathrm{P}$ was $0 \mathrm{~cm}$ (5th-95th percentile; $0-5.7 \mathrm{~cm})$ and at $\mathrm{D}$ the median was $0 \mathrm{~cm}(5 \mathrm{th}-95$ th percentile; $0-6.0 \mathrm{~cm})$. Paralleling the increasing dimensions of the pressure troughs, the magnitude of the distal contractile 
integral for the segment between $\mathrm{P}$ and D progressively decreased from 1,385 $\mathrm{mm} \mathrm{Hg-s-cm}$ for the median EPT plot to $996 \mathrm{~mm} \mathrm{Hg}-\mathrm{s}-\mathrm{cm}$ for the 25th percentile plot and $363 \mathrm{~mm} \mathrm{Hg}$-s$\mathrm{cm}$ for the 5 th percentile plot.

\section{Peristaltic integrity in dysphagia patients compared with normal subjects}

To summarize, HRIM data suggest that failed peristalsis is uniformly associated with IBT and when $20 \mathrm{~mm} \mathrm{Hg}$ isobaric contour breaks exceed $5 \mathrm{~cm}$ in length they also will uniformly be associated with IBT; when breaks are in the range of $2-5 \mathrm{~cm}$ they will variably be associated with IBT. These findings were observed to some extent in the 75 control subjects. Given these data, the frequency of occurrence of these three phenomena (failed peristalsis, large breaks in the $20 \mathrm{~mm} \mathrm{Hg}$ isobaric contour, and small breaks in the $20 \mathrm{~mm} \mathrm{Hg}$ isobaric contour) was each compared between the control subjects and dysphagia patients to see which, if any, correlated with non-obstructive dysphagia.

Table 2 indicates the normal limits for each of the EPT defects potentially indicative of impaired peristalsis in the 75 control subjects and compares the excessive occurrence of each in patients with unexplained dysphagia to that of control subjects. Large and small breaks in the $20 \mathrm{~mm} \mathrm{Hg}$ isobaric contour, but not failed peristalsis, were significantly more common in the dysphagia patients. The limits of failed peristalsis in the dysphagic patients (median $0 \%$, 95th percentile $44 \%$ ) were similar to that in control subjects (median $0 \%$, 95th percentile $30 \%$ ). As was observed in the process of constructing the normalized EPT in which each subject exhibited a stereotyped EPT pattern, such was also true for defects in EPT with each subject repetitively demonstrating a particular pattern. Figure 6, illustrates that, although some overlap among diagnoses existed, 38 dysphagic patients met only one of these criteria of impaired peristalsis, 8 met two criteria, and no patient exhibited all three.

The data in Table 2 suggest that, although both are associated with IBT, failed peristalsis and frequent breaks in the $20 \mathrm{~mm} \mathrm{Hg}$ isobaric contour should be considered independently because they were usually independent findings and only the latter was shown to correlate with unexplained non-obstructive dysphagia. Consequently, the categorization summarized in Table 3 is proposed. Note that individuals with absent peristalsis (100\% failed peristalsis) were excluded from the current study but are included in Table 3 as a unique entity of clear significance.

\section{DISCUSSION}

The major aims of this investigation were (i) to establish normal limits of peristaltic integrity in EPT terms based on a systematic analysis of a large series of control subjects; and (ii) to develop a classification scheme for weak peristalsis based on a comparison between control subjects and a cohort of patients with unexplained non-obstructive dysphagia intended for use in clinical EPT studies. The major findings were that the segmental architecture of peristalsis was highly stereotyped among subjects as were defects in that architecture associated with IBT for individual subjects: large $(>5 \mathrm{~cm})$ and small $(2-5 \mathrm{~cm})$ breaks in the $20 \mathrm{~mm} \mathrm{Hg}$ isobaric contour of the peristaltic contraction. Although encountered in only about one-third of the 113 patients studied, frequent large and small breaks in the $20 \mathrm{~mm} \mathrm{Hg}$ isobaric contour were significantly more common in the dysphagia patients than in control subjects. Failed peristalsis, the other mechanism of IBT observed in the HRIM studies occurred no more frequently in the dysphagia population than in the control subjects. Based on these observations, we propose an EPT classification of weak peristalsis based on the occurrence of breaks in the $20 \mathrm{~mm} \mathrm{Hg}$ isobaric contour wherein weak peristalsis with large breaks is defined by those occurring with $>20 \%$ of swallows and weak peristalsis with small breaks defined by those occurring with $>30 \%$ of swallows. 
A common indication for esophageal function testing is in the evaluation of unexplained dysphagia. Findings of indisputable significance are the detection of major motor disorders or absent peristalsis. However, those findings are relatively uncommon. More frequent is the finding of hypotensive or mildly disordered peristalsis, the significance of which is less certain $(14,15)$. Acknowledging that caveat, IBT seems a reasonable surrogate end point for gauging the adequacy of peristalsis. IBT occurs more frequently with weak peristalsis. Kahrilas et al. (3) reported that IBT invariably occurred in distal esophagus when peristaltic amplitude was $<20 \mathrm{~mm} \mathrm{Hg}$, whereas it rarely occurred when the peristaltic amplitude was $31-45 \mathrm{~mm} \mathrm{Hg}$. Moreover, IBT is associated with dysphagia. In a study of 350 patients, IBT occurred in $51 \%$ of patients with dysphagia compared with $30 \%$ of patients without dysphagia (6). Consequently, we evaluated EPT defects associated with IBT in HRIM studies as potentially linked to dysphagia. Consistent with previous investigations $(7,10,16)$, we found that failed peristaltic contractions and EPT plots with breaks in the $20 \mathrm{~mm} \mathrm{Hg}$ isobaric contour were associated with IBT. EPT plots with breaks $>5 \mathrm{~cm}$ (large) were nearly uniformly associated with IBT; $2-5 \mathrm{~cm}$ (small) breaks were variably associated with IBT (Table 1). Topography plots without breaks or with breaks in the $20 \mathrm{~mm} \mathrm{Hg}$ isobaric contour $<2 \mathrm{~cm}$ in length uniformly achieved complete bolus transit.

Previous impedance manometry studies found that $30 \%$ of swallows with IBT to be the upper limit of normal $(10,17,18)$. The normative data in Table 2 generally support that finding. However, we found that although small and large breaks in $20 \mathrm{~mm} \mathrm{Hg} \mathrm{IBC}$ occurred more frequently in the dysphagic patients than in the control subjects, this was not so for failed peristalsis. A caveat to this is that patients with absent peristalsis (100\% failed) were considered to have a major motility disturbance and excluded from this analysis at the outset. Consequently, in the proposed classification, frequently failed peristalsis was considered as its own entity and not included as an element in definition of weak peristalsis (Table 3).

The finding that frequent large or small breaks in the $20 \mathrm{~mm} \mathrm{Hg} \mathrm{IBC} \mathrm{occurred} \mathrm{significantly}$ more frequently in a carefully selected patient population with unexplained dysphagia suggests that this mechanism may explain dysphagia in some patients with normal esophagogastric junction relaxation, no hiatal hernia, no stricture or ring, no finding suggestive of eosinophilic esophagitis, and no major motility disorder. Although this was possibility suggested in a previous case report (7) and in an analysis of transition zone defects (18), this is the first study to our knowledge that systematically evaluated these phenomena in a large cohort of patients with unexplained dysphagia.

This study has limitations, one of which is potential age bias between control subjects (mean 27 years, range 19-48 years) and dysphagia patients (mean 46 years, range $21-76$ years). Subdividing the dysphagia group into those younger or older than 45 years, we found no significant age effect, but the possibility of bias remains, and including older control subjects into a subsequent analysis could potentially increase the number of swallows with breaks in the $20 \mathrm{~mm} \mathrm{Hg}$ isobaric contour defining weak peristalsis. Another potential limitation of the study was that the HRIM analysis was based on only 16 normal subjects studied with the Sierra HRIM device. However, the HRIM data were largely confirmatory of previous results obtained with another HRIM system (Medical Measurements Systems, Enschede, The Netherlands) in a similar analysis of another 24 subjects (10). Finally, the use of provocative testing with solid or volume challenge in correlation with symptom assessment may be shown to improve the diagnostic yield of EPT studies in the future (19).

In summary, we utilized a large set of control subjects to define the stereotypic features of EPT common among subjects and then to define normal limits for EPT features associated with IBT as verified by HRIM. The defining features of weak peristalsis (Table 3), frequent 
large or small breaks in the $20 \mathrm{~mm} \mathrm{Hg}$ isobaric contour, occurred significantly more frequent in dysphagia patients than in control subjects making this a potential explanation for unexplained dysphagia. The therapeutic implications of these findings, both with respect to tolerance of antireflux surgery and responsiveness to pharmacological intervention with pro-motility drugs will need to be the focus of future research.

\section{Study Highlights \\ WHAT IS CURRENT KNOWLEDGE}

- Esophageal peristaltic amplitudes $>30 \mathrm{~mm} \mathrm{Hg}$ are normally associated with complete bolus transit, but impedance studies suggest that this threshold poorly predicts incomplete bolus transit (IBT).

- Esophageal pressure topography (EPT) studies demonstrate a segmental architecture of peristalsis characterized by non-uniform peristaltic amplitude with discrete segments of contraction and intervening pressure troughs.

- High-resolution impedance manometry shows that IBT occurs at pressure troughs in EPT plots and with failed peristalsis.

\section{WHAT IS NEW HERE}

- Computer simulation was used to extract the stereotypic features of EPT plots in control subjects and establish normal limits for failed peristalsis and for the dimensions and occurrence of critical pressure troughs in peristalsis.

- Large (> $5 \mathrm{~cm})$ and small $(2-5 \mathrm{~cm})$ pressure troughs in the $20 \mathrm{~mm} \mathrm{Hg}$ isobaric contour of peristalsis, but not failed peristalsis, occurred more frequently in patients with unexplained non-obstructive dysphagia than in control subjects.

- A classification scheme for peristaltic integrity is proposed in EPT terms establishing quantitative and qualitative limits for pressure troughs and failed peristalsis intended for use in clinical EPT studies.

\section{Acknowledgments}

Financial support: This work was supported by R01 DK56033 (P.J.K.) and R01 DK079902 (J.E.P.) from the Public Health Service.

\section{References}

1. Leite LP, Johnston BT, Barrett J, et al. Ineffective esophageal motility (IEM): the primary finding in patients with nonspecific esophageal motility disorder. Dig Dis Sci 1997;42:1859-65. [PubMed: 9331148]

2. Spechler SJ, Castell DO. Classification of oesophageal motility abnormalities. Gut 2001;49:145-51. [PubMed: 11413123]

3. Kahrilas PJ, Dodds WJ, Hogan WJ. Effect of peristaltic dysfunction on esophageal volume clearance. Gastroenterology 1988;94:73-80. [PubMed: 3335301]

4. Sifrim D, Silny J, Holloway RH, et al. Patterns of gas and liquid reflux during transient lower oesophageal sphincter relaxation: a study using intraluminal electrical impedance. Gut 1999;44:4754. [PubMed: 9862825]

5. Clouse RE, Staiano A. Topography of normal and high-amplitude esophageal peristalsis. Am J Physiol 1993;265:G1098-107. [PubMed: 8279561]

6. Tutuian R, Castell DO. Combined multichannel intraluminal impedance and manometry clarifies esophageal function abnormalities: study in 350 patients. Am J Gastroenterol 2004;99:1011-9. [PubMed: 15180718] 
7. Fox M, Hebbard G, Janiak P, et al. High-resolution manometry predicts the success of oesophageal bolus transport and identifies clinically important abnormalities not detected by conventional manometry. Neurogastroenterol Motil 2004;16:533-42. [PubMed: 15500509]

8. Kahrilas PJ, Clouse RE, Hogan WJ. American Gastroenterological Association technical review on the clinical use of esophageal manometry. Gastroenterology 1994;107:1865-84. [PubMed: 7958705]

9. Pandolfino JE, Kahrilas PJ. AGA technical review on the clinical use of esophageal manometry. Gastroenterology 2005;128:209-24. [PubMed: 15633138]

10. Bulsiewicz WJ, Kahrilas PJ, Kwiatek MA, et al. Esophageal pressure topography criteria indicative of incomplete bolus clearance: a study using high-resolution impedance manometry. Am J Gastroenterol 2009;104:2721-8. [PubMed: 19690527]

11. Pandolfino JE, Fox MR, Bredenoord AJ, et al. High-resolution manometry in clinical practice: utilizing pressure topography to classify oesophageal motility abnormalities. Neurogastroenterol Motil 2009;21:796-806. [PubMed: 19413684]

12. Pandolfino JE, Leslie E, Luger D, et al. The contractile deceleration point: an important physiologic landmark on oesophageal pressure topography. Neurogastroenterol Motil 2010;22:395-400. [PubMed: 20047637]

13. Kahrilas PJ, Lin Z, Kwiatek M, et al. Normalized high resolution esophageal pressure topography (HREPT) data: characterizing peristalsis in terms of neuromuscular physiology. Gastroenterology 2010;138:S604-5.

14. Imam H, Shay S, Ali A, et al. Bolus transit patterns in healthy subjects: a study using simultaneous impedance monitoring, videoesophagram, and esophageal manometry. Am J Physiol Gastrointest Liver Physiol 2005;288:G1000-6. [PubMed: 15826930]

15. Nguyen NQ, Rigda R, Tippett M, et al. Assessment of oesophageal motor function using combined perfusion manometry and multi-channel intraluminal impedance measurement in normal subjects. Neurogastroenterol Motil 2005;17:458-65. [PubMed: 15916634]

16. Ghosh SK, Janiak P, Fox M, et al. Physiology of the oesophageal transition zone in the presence of chronic bolus retention: studies using concurrent high resolution manometry and digital fluoroscopy. Neurogastroenterol Motil 2008;20:750-9. [PubMed: 18422907]

17. Tutuian R, Vela MF, Balaji NS, et al. Esophageal function testing with combined multichannel intraluminal impedance and manometry: multicenter study in healthy volunteers. Clin Gastroenterol Hepatol 2003;1:174-82. [PubMed: 15017488]

18. Tutuian R, Elton JP, Castell DO, et al. Effects of position on oesophageal function: studies using combined manometry and multichannel intraluminal impedance. Neurogastroenterol Motil 2003;15:63-7. [PubMed: 12588470]

19. Sweis R, Anggiansah R, Wong T, et al. High resolution manometry with large volume multiple repeated swallows aids the detection of esophageal pathology. Gastroenterology 2008;134:A719. 


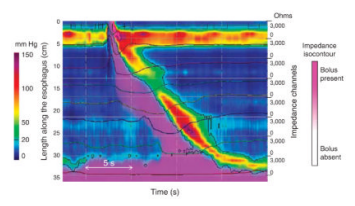

Figure 1.

Example of intact peristalsis associated with complete bolus transit in high-resolution impedance manometry (HRIM) study. Impedance tracings are superimposed to esophageal pressure topography (EPT) and impedance data are also displayed by overlaid pink colorization. The pink shaded area indicates bolus presence. Complete bolus transit was observed with the onset of contraction at each esophageal level corresponding to the clearance of pink colorization and the upward inflection in the impedance tracings. 


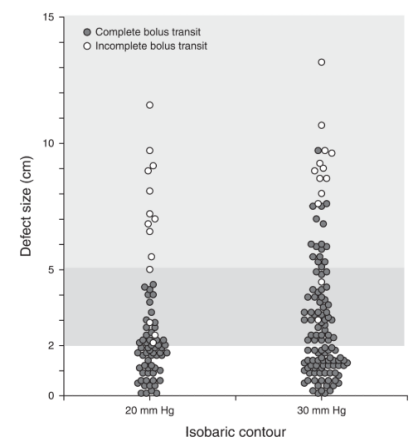

\section{Figure 2.}

Bolus transit as a function of 20 and $30 \mathrm{~mm} \mathrm{Hg}$ isobaric contour integrity. Incomplete bolus transit (IBT) was uniformly associated with $\geq 2 \mathrm{~cm}$ breaks in the $20 \mathrm{~mm} \mathrm{Hg}$, but not $30 \mathrm{~mm}$ $\mathrm{Hg}$, isobaric contour. Breaks $>5 \mathrm{~cm}$ in the $20 \mathrm{~mm} \mathrm{Hg}$, but not $30 \mathrm{~mm} \mathrm{Hg}$, isobaric contour (light gray shading), were uniformly associated with IBT. Small breaks of $2-5 \mathrm{~cm}$ in length (darker shading) were variably associated with IBT. Consequently, optimal discrimination between complete and incomplete bolus transit was achieved utilizing the $20 \mathrm{~mm} \mathrm{Hg}$ isobaric contour making this the superior metric. Note that complete bolus transit, as seen on impedance, that was associated with intact esophageal pressure topography at $20 \mathrm{~mm} \mathrm{Hg}(n$ $=62)$ and $30 \mathrm{~mm} \mathrm{Hg}$ isobaric contour $(n=16)$ is not displayed on the figure. 


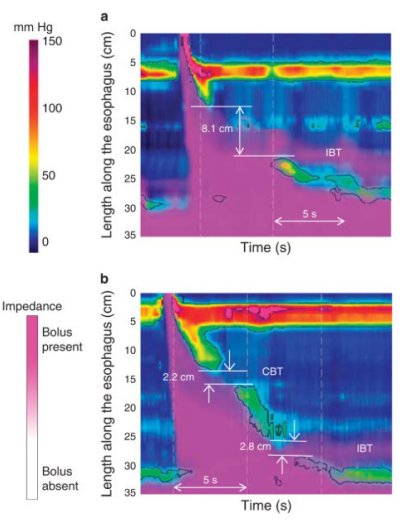

Figure 3.

Examples of incomplete bolus transit (IBT) in high-resolution impedance manometry (HRIM) studies attributable to large or small breaks in the $20 \mathrm{~mm} \mathrm{Hg}$ isobaric contour. Impedance data are displayed by overlaid pink colorization with the pink shading indicative of areas on the topography plots with retained bolus. The associated dimensions of the critical break in the isobaric contours are indicated on each panel. In the panel a, IBT is associated with a large proximal break in the $20 \mathrm{~mm} \mathrm{Hg}$ isobaric contour. In panel b, the small proximal break is associated with complete bolus transit, whereas the distal break is associated with IBT. 


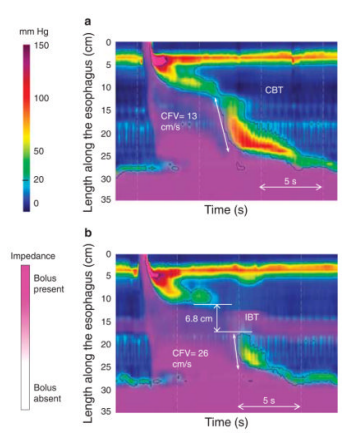

Figure 4.

Two esophageal pressure topography (EPT) plots with rapid contractile front velocity (CFV) from the same subject. In panel a, there is complete bolus transit and no break in the $20 \mathrm{~mm}$ $\mathrm{Hg}$ isobaric contour. In panel $\mathbf{b}$ there is incomplete bolus transit (IBT) and a large break in the $20 \mathrm{~mm} \mathrm{Hg}$ isobaric contour. CFV had no impact on bolus transit in the absence of a concomitant defect in the $20 \mathrm{~mm} \mathrm{Hg}$ isobaric contour. 

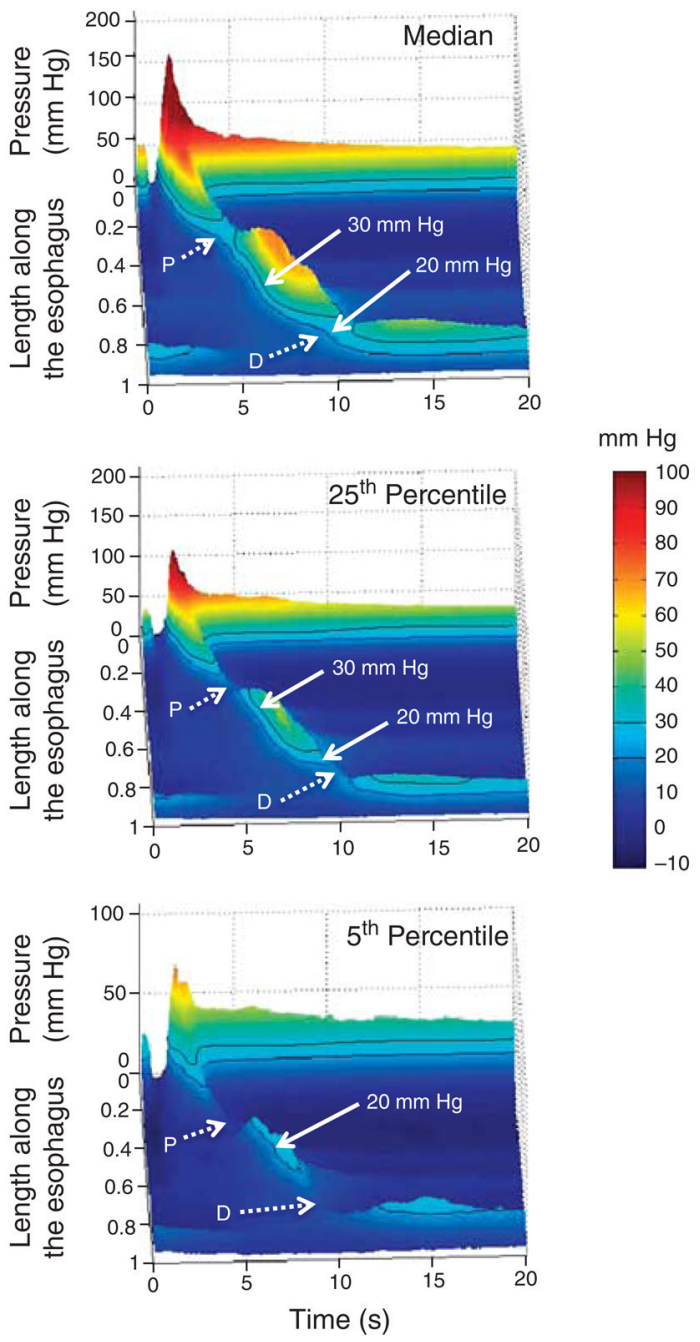

Figure 5.

The median composite esophageal pressure topography (EPT) landscape plot from 75 subjects is shown on the top panel. The proximal (P) and distal (D) pressure troughs are indicated with dashed arrows. The corresponding 25 th and 5 th percentile EPT composites are illustrated in the middle and bottom panels. Note that although the 20 and $30 \mathrm{~mm} \mathrm{Hg}$ isobaric contours are seamless in the median (50th percentile) swallow, these acquire progressively lengthier breaks in the 25 th and 5 th percentile composites, respectively. 


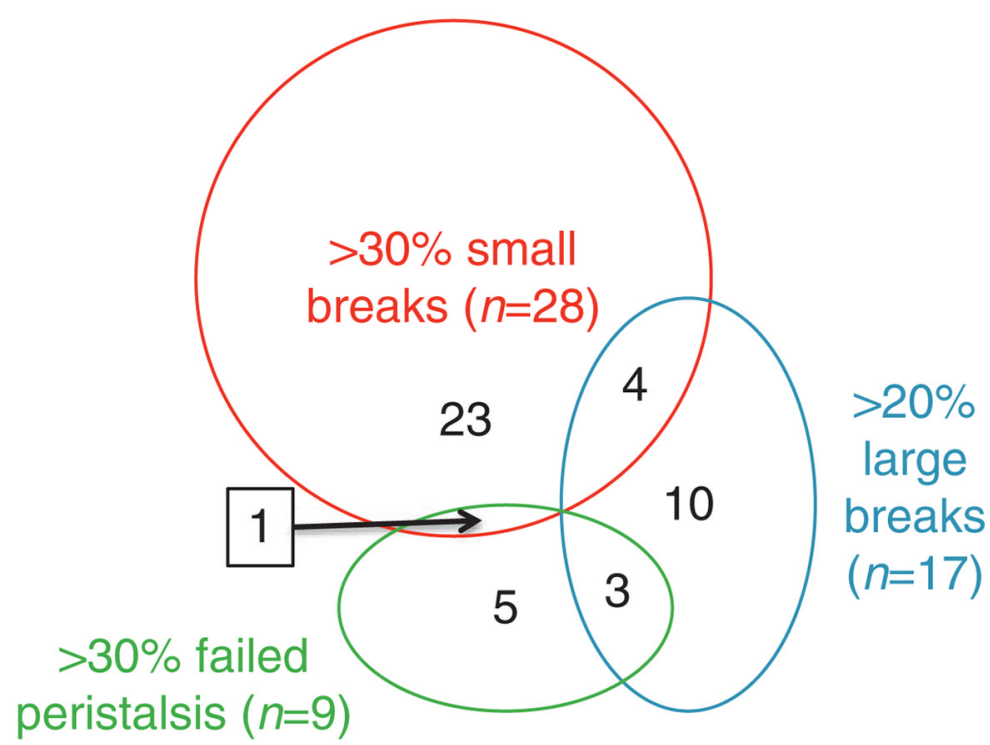

Figure 6.

Venn diagram illustrating the extent of overlap between dysphagic patients with frequent failed peristalsis (green circle), > $30 \%$ of swallows with small breaks (red circle), and > 20 $\%$ of swallows with large breaks in the $20 \mathrm{~mm} \mathrm{Hg}$ isobaric contour (blue circle). 
Table 1

Bolus transit in HRIM studies with peristaltic contractions characterized by the integrity of the $20 \mathrm{~mm} \mathrm{Hg}$ isobaric contour

\begin{tabular}{|c|c|c|}
\hline & Incomplete bolus transit, $n(\%)$ & Complete bolus transit, $n(\%)$ \\
\hline Failed peristalsis & $13(100)$ & $0(0)$ \\
\hline \multicolumn{3}{|c|}{$20 \mathrm{~mm} \mathrm{Hg}$ isobaric contour integrity } \\
\hline No break & $0(0)$ & $62(100)$ \\
\hline$<2 \mathrm{~cm}$ break & $0(0)$ & $50(100)$ \\
\hline $2-5 \mathrm{~cm}$ break & $\begin{array}{l}4(16) \\
2 \mathrm{P}, 2 \mathrm{D}\end{array}$ & $\begin{array}{c}21(84) \\
13 \mathrm{P}, 5 \mathrm{M}, 3 \mathrm{D}\end{array}$ \\
\hline$>5 \mathrm{~cm}$ break & $\begin{array}{l}10(100) \\
8 \mathrm{P}, 2 \mathrm{D}\end{array}$ & $0(0)$ \\
\hline Total, $n$ & 27 & 133 \\
\hline
\end{tabular}

HRIM, high-resolution impedance manometry.

Breaks were subcharacterized by location as proximal (P), middle (M), or distal (D). 


\section{Table 2}

The frequency of occurrence of pressure topography features associated with IBT among 75 control subjects and 113 patients with dysphagia

\begin{tabular}{|lcccc|}
\hline & $\begin{array}{c}\text { Normal range (0-95th } \\
\text { percentile, 75 control } \\
\text { subjects) (\%) }\end{array}$ & \multicolumn{2}{c|}{ Number of subjects exceeding the 95th percentile of normal } \\
\cline { 3 - 6 } Pressure topography defect & $0-30$ & Control subjects, $\boldsymbol{n}(\boldsymbol{\%})$ & $\begin{array}{c}\text { Dysphagia patients, } \boldsymbol{n} \\
(\boldsymbol{\%})\end{array}$ & $\boldsymbol{P}$ value $\boldsymbol{a}$ \\
\hline Failed peristalsis & $0-20$ & $3(4)$ & $9(8)$ & 0.54 \\
\hline $\begin{array}{l}\text { Large breaks in 20 mm Hg isobaric } \\
\text { contour }\end{array}$ & $0-30$ & $\begin{array}{c}3(4) \\
\text { 2P, 0M, 1D }\end{array}$ & 13P, 3M, 1D & 0.02 \\
\hline $\begin{array}{l}\text { Small breaks in } 20 \mathrm{~mm} \text { Hg isobaric } \\
\text { contour }\end{array}$ & & $\begin{array}{c}3(4) \\
\text { 2P, 1M, 0D }\end{array}$ & 24P, 2M, 2D & $<0.01$ \\
\hline
\end{tabular}

IBT, incomplete bolus transit.

${ }^{a}$ Control subjects vs. dysphagia patients, chi-square test.

Large (> $5 \mathrm{~cm}$ ) and small $(2-5 \mathrm{~cm})$ defects in the $20 \mathrm{~mm} \mathrm{Hg}$ isobaric contour were localized to the proximal (P), middle (M), or distal (D) pressure troughs. 


\section{Table 3}

Proposed classification of peristaltic integrity in EPT

\begin{tabular}{|ll|}
\hline Diagnosis & Diagnostic criteria (all with normal esophago-gastric junction relaxation) \\
\hline Absent peristalsis & $100 \%$ of swallows with failed peristalsis \\
\hline Frequent failed peristalsis $a$ & $>30 \%$, but $<100 \%$ of swallows with failed peristalsis \\
\hline Weak peristalsis with large peristaltic defects & $>20 \%$ of swallows with $>5 \mathrm{~cm}$ breaks in the $20 \mathrm{~mm} \mathrm{Hg}$ isobaric contour \\
\hline Weak peristalsis with small peristaltic defects & $>30 \%$ of swallows with $2-5 \mathrm{~cm}$ breaks in the $20 \mathrm{~mm} \mathrm{Hg}$ isobaric contour \\
\hline
\end{tabular}

EPT, esophageal pressure topography.

${ }^{a}$ Although statistically exceeding the 95th percentile of normal, this finding has not been shown to correlate with non-obstructive dysphagia.

Isobaric contour pressure is referenced to atmospheric. Note that an individual may have more than one diagnosis 